\title{
Service Routing Analysis in Optical Network with Algebraic Decision Diagram
}

\author{
Zhimin Xiang ${ }^{a^{*}}$, Yongwei Chen ${ }^{b}$, Jin Liu $^{c}$ and Xiaoxiao Wo ${ }^{d}$ \\ State Grid Information\&Communication Company of Hunan Electric Power Corporation, Changsha, \\ Wuhan 410007, China \\ axiangzm511@sina.com, ${ }^{b}$ cyw70@sina.com, ${ }^{\mathrm{C}}$ liujin518@yeah.net, ${ }^{\mathrm{d}}$ woxix@sina.com
}

\begin{abstract}
Keywords: Routing, Optical network, Wavelength converter.
Abstract. The existing research results show that in the network configuration section WC can get close to the performance of the whole wavelength conversion network. The design of optical network backbone should not only satisfy the existing service, but also consider further involved service in the electric power system. Partial wavelength conversion capability of sparse wavelength is temporarily an intermediate form during the evolution of power system communication system. This paper mainly studied the WC in the sparse wavelength conversion network configuration and routing algorithm and wavelength assignment, and we have proposed a new multi-layer wavelength assignment algorithm by using multi-layer wavelength division. The results show that in the sparse wavelength conversion network, the network performance and routing wavelength assignment algorithm, the number of wavelength converter and the position of the wavelength converter has a direct relationship.
\end{abstract}

\section{Introduction}

At present, the optical transmission network technology has been widely commercialized in the electric power communication network. High bandwidth optical transport network is preferred to meet the bandwidth requirement of electric power communication network is growing. Even in the modern power system communication network, not all nodes are equipped with wavelength conversion capability. In order to achieve the establishment of the optical channel, all service flow must be all assigned with the same link free wavelength optical channel. The node configuration wavelength converter (WC) can make the light channel without being limited by the wavelength of uniform continuity, and it can effectively reduce the network model complexity as mentioned in [1]. Because the price of the WC is more expensive, for all nodes in the network configuration WC would increase the cost of network construction and maintenance, given the current state between the average capacities of the backbone network connectivity is low, only for parts of the network node configuration. By using wavelength converter, the operator can reduce the network's overall investment ${ }^{[2]}$. The existing research results show that in the network configuration section WC can get close to the performance of the whole wavelength conversion network. When making its optical backbone network design, should not only satisfy the existing business, and aspires to future business with the development of the network. As the future electric power system service requirement is not as clear as the telecommunication system, it can temporarily by configuring with partial wavelength conversion capability of sparse wavelength conversion network networking.

Along with the development of smart grid construction and modernization of power grid enterprises, electric power business application will generally present IP, the trend of the broadband, video. The traditional electric power communication network leading technology for SDH, therefore, carry business will lead to transfer IP network together with function change. In addition, the granularity in electric power communication system is quite different, i.e., dispatching system, protection and fault information system, energy management system, etc. At present, in addition to the power production control in electric power optical fiber private network business is given priority to with $2 \mathrm{Mb} / \mathrm{s}$ and $155 \mathrm{Mb} / \mathrm{s}$. With the development of the business in electric power system, the business of small particles, $2.5 \mathrm{~Gb} / \mathrm{s}$ and $10 \mathrm{~Gb} / \mathrm{s}$ business will be widely applied. The proportion of $40 \mathrm{~Gb} / \mathrm{s}$ will in future possible business. IP service oriented architecture, and a new generation of 
electric power transmission network overall trend from SDH to its OTN evolution, bearing large particles directly by IP business is how to build a low cost efficient short hand. How in the limited resource in the network for the business to choose the appropriate routing and wavelength allocation optimization for the use of network resources and the management and control has great influence. So, the wavelength assignment problem becomes one of the core issues of optical transport network.

This paper mainly studied the WC in the sparse wavelength conversion network configuration and routing and wavelength assignment (RWA) problem, to reach the dynamic business looking for routing and distribution channel resources, with the help of the layered graph model routing and wavelength assignment can be done at the same time, and in the layered graph RWA problem can be converted to find the shortest path problem between the source and destination node. In view of the four classic WC configuration algorithm, this paper for the sparse wavelength conversion network routing strategy based on hierarchical graph model is proposed. Optical wavelength converters are introduced to different links on the same channel can use different wavelengths of establishing business connections, through virtual wavelength path narrow wavelength limit. In this paper, through constructing optical backbone network simulation platform to verify the blocking performance, analysis of WC number configuration, the influence of the routing strategy on network backbone. The experimental results show that the network blocking performance has close relation with WC allocation approach and the routing policy.Please keep a second copy of your manuscript in your office. When receiving the paper, we assume that the corresponding authors grant us the copyright to use the paper for the book or journal in question. Should authors use tables or figures from other Publications, they must ask the corresponding publishers to grant them the right to publish this material in their paper.

\section{RWA Algorithms under different WC deployment}

\section{Problem Illustration}

Optical transport network design usually considers the connection of electric cross connect layer, which involves the logical topology of the network. Therefore, the construction of underlying optical transport network shall consider the actual physical topology and service flow in electric power system $^{[3,4]}$. Literature [5] presents the wavelength converter configuration and deployment approach on Mesh network, and in which the virtual wavelength path is quite convenient for the service flow route computation. The optimized configuration of wavelength converter is refers to under the condition of a given network topology and link capacity, how to put the wavelength converter makes network model, and then the lowest cost can be obtained. Among several typical algorithms, the ADD (Algebraic Decision Diagram) is widely used in the optical network, and it is also quite convenient for the application in electric power system communication network.

The basic idea of Sub_ADD algorithm is the concept of using auxiliary subgraph accelerating ADD algorithm. ADD general idea of the algorithm is first to unified numbering of all nodes, and then according to the node number sequence will not with wavelength conversion capability node in the network preconfigured, calculate each preconfigured network model and then compared with the concluded that the minimum value. First Fit (FF), Least Use (LU) and Most Used (MU) algorithms are the most common wavelength assignment algorithm. The WC configuration strategy can be implemented in the preconfigured node. Sub_ADD algorithm computation is quite complex and can be accelerated with the auxiliary orientation.

\section{Fabrication of Waveband sharing WC}

In fabrication of WC is shown in fig.1, with full range tunable wavelength converter (FRTWC) configuration method is the typical method of waveband sharing configuration. The switching fabric that using FRTWC can be used to solve the problem of light path establishing, and the configuration of FRTWC sharing pool, in the form of feedback is shared by all of the input port. Therefore, the configured FRTWC can be made full use of the common buffer. In the shared pool FRTWC is divided into $\mathrm{M}$ groups, each group has $\mathrm{W}$ input channels. FRTWC, with corresponding to the $\mathrm{W}$ input 
wavelength channels. When light path group competition occurs, only one group of light can carry on the output to the wavelength of the original, other light group as far as possible can be used to solve the competition problem using wavelength converter. At this point, if there are other free wavelength output ports, the corresponding wavelength converter in FRTWC shared pool is free. The light of the competitive group can exchange first to FRTWC wavelength conversion in the shared pool, and then through the exchange of matrix switching to the output port of destination free wavelength. If you can't use FRTWC to tackle the competition, competition of optical packet will be discarded.

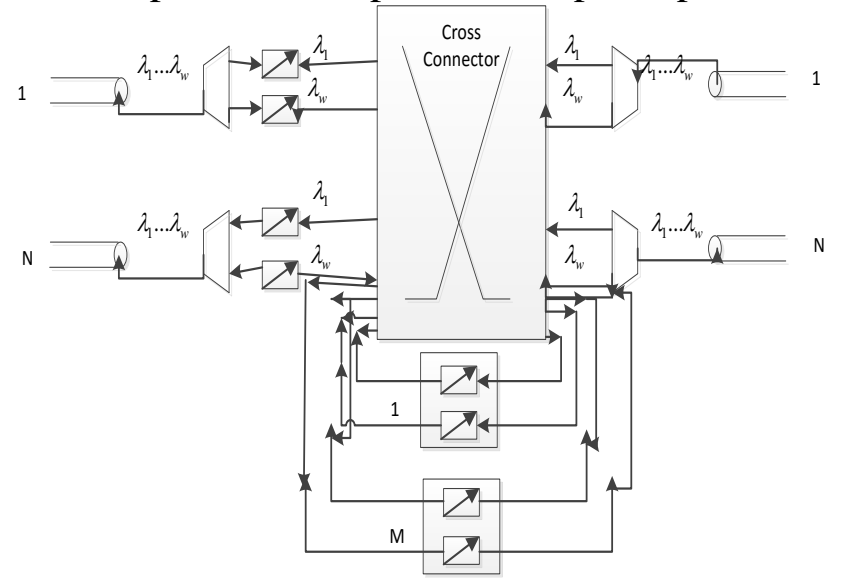

Fig.1 Waveband sharing WC cross connector

In auxiliary subgraph, the simplified network topology only includes the path from root node to destination node, and in addition to all other nodes is the source node. As a result, the service request always randomly generated in the root node, and call the destination node is always the root node. Whether a network is configured with a wavelength converter, it can be distinguished from external point and node pair configuration diagram. This is because the graph of a path cannot be obtained from the wavelength converter conversion gain. If a network is assumed that have node wavelength conversion capability node, it is obviously that this will affect the topology. Only when a wavelength converter configuration to the internal point subgraph, we just need to calculate, this is because there is always a child node will benefit from this configuration. Sub_ADD algorithm is based on the graph above nature successfully accelerated the ADD algorithm.

\section{Multilayer wavelength assignment strategy}

Dynamic RWA problem in networks without wavelength conversion, has been widely studied, including hierarchical Graph (Layered Graph, LG) model is a one-off the best way to solve the problem of RWA. Hierarchical graph model applies to sparse wavelength conversion network. The physical topology is modeled as $\mathrm{G}(\mathrm{N}, \mathrm{L}, \mathrm{W})$, where $\mathrm{N}, \mathrm{L}, \mathrm{W}$ denote the number of node set, link set, and available optical fiber wavelength set respectively. Assume that each link is composed of a pair of opposite direction of unidirectional fiber, each fiber wavelength set \{available lambda 1, lambda. $2, \ldots$ Lambda W\}. Hierarchical graph model is actually a graph extending technology by using copied network physical topology. During over layered network, each layer corresponds to a wavelength plane. Sparse wavelength conversion in the network layered graph model as shown in the following figure. In which node A have wavelength conversion capability. The RWA problem is simplified to the single layer or layers to find the optimal path from source node to destination node problem, namely how to take the routing strategy to find the optimal routing between each layer.

Sub_ADD algorithm is described in detail as follows:

Step1: Construct N-node auxiliary directed graph and internal nodes of each subgraph is denoting as set $\{\mathrm{I}\}$;

Step2: WC is deployed in this step. Set the current iteration for the first iteration, and configure the $\mathrm{k}$-th wavelength converter according to the following steps to configure the network node: 
1) Select $\mathrm{N}-\mathrm{k}+1$ node that are not configured wavelength converter, and assigned a number $\mathrm{j}$. Considering the pre-configured nodes that have wavelength conversion capability, and then according to the network configuration of the wavelength converter at this time of $\mathrm{N}$ subgraph mode and then compute each block probability respectively:

(a) Set Node $\mathrm{j}$ as WC node;

(b) For subgraph $\mathrm{Gi}(1<\mathrm{i}<\mathrm{N})$, check whether the node $\mathrm{j}$ is the internal nodes of the subgraph $\mathrm{Gi}$, If yes, recalculate the subgraph Gi connectivity in node j. Otherwise, return to the mail loop;

(c) If $1<\mathrm{i}<\mathrm{N}$, let $\mathrm{i}=\mathrm{i}+1$ and jumps to step (b). Otherwise, recalculate the network global connectivity rate with node $\mathrm{j}$.

2) if $1 \leq \mathrm{j}<\mathrm{N}$, then let $\mathrm{j}=\mathrm{j}+1$ and repeat step 1 ). Otherwise, analyze the maximum connectivity rate in iteration. Confirm the k-th WC that will make the network with largest connectivity.

Step3: If $1 \leq \mathrm{k}<\mathrm{K}$, then let $\mathrm{k}=\mathrm{k}+1$, jump to step1. Otherwise, the algorithm is over.

According the above steps, we can obtained that in Step2, there is about N(N-1) path that need to be computed for the connectivity. After the k-th WC configuration, the maximum path that need to be computing is about $\mathrm{N}(\mathrm{N}-1) *(\mathrm{~N}-\mathrm{k}+1)$. Then, we can conclude that if $\mathrm{K}$ converter is included, the $\mathrm{k}-\mathrm{th}$ WC will influence ${ }^{N(N-1) \sum_{k=1}^{K}(N-k+1)}$ paths. The advantage of this algorithm is quite obvious for a higher percentage of internal nodes $\{\mathrm{I}\}$.

According to the configuration principle of ADD algorithm, the preconfigured network will obtain a maximum connectivity. According to statistics, the wavelength converter configuration connection rate is higher on the node in the network, network configuration is complete. The multilayer wavelength assignment algorithm using auxiliary directed graph will accelerate the computation speed. The proposed algorithm model of external node configuration wavelength converter is also unable to get the conversion gain, and therefore external node does not participate in the iterative process configuration.

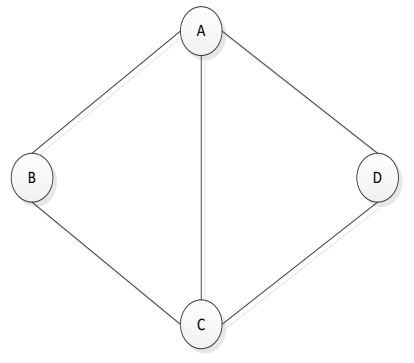

(a) Physical topology with four nodes

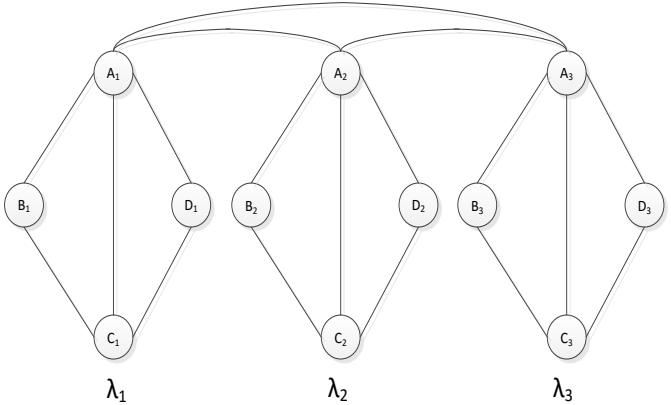

(b) Multilayer wavelength plane division Fig.2 Multilayer network division with sparse WC configurations

\section{Experimental results}

In order to illustrate the effectiveness of proposed algorithm, we use the following high-capacity backbone optical transmission as demonstration (48 nodes, 64 links) channel allocation simulation calculation. Assume that each link of the wave number $F=8$. The optimization object is to minimize the use of wavelength capacity. The results shown in the following table, use the wavelength of the capacity is 129 in total.

In order to illustrate the performance we have applied several algorithms for comparison. Considering the possibility of blocking the greater the degree of node in the network, the greater the possible wavelength converter placed in a large degree on the node is an important way to reduce network complexity (especially when the network load increase). As in the optical network, longer path length will always experience higher risk of network congestion. Take the constraint of a quarter of WC configuration, and then we obtain the following result for $\mathrm{WC}$ deployment. The detail deployment of WC place is presented in Table I. 


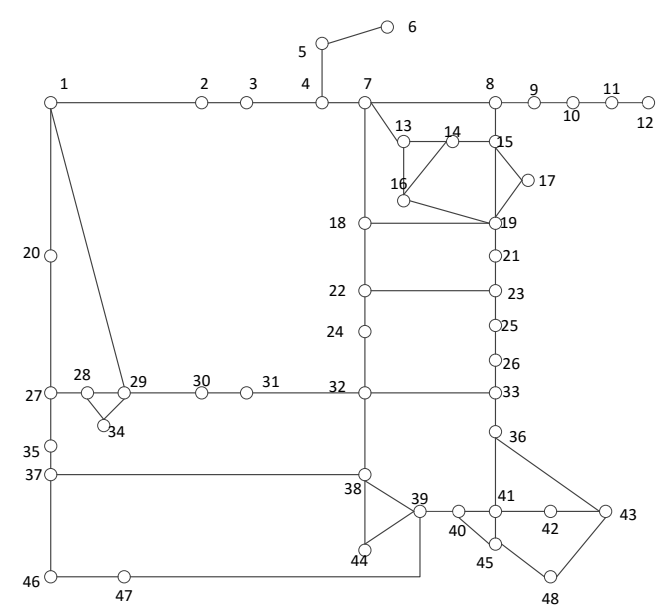

Fig.3 48-nodes electric power communication system backbone

\begin{tabular}{|c|c|}
\hline Algorithm & WC Configuration Results \\
\hline Sub+ADD & $\begin{array}{c}32,7,1,22,33,37,9,3,23,39, \\
30,19\end{array}$ \\
\hline $\begin{array}{c}\text { Proposed } \\
\text { Algorithm }\end{array}$ & $\begin{array}{c}32,7,1,22,33,37,9,3,23,39, \\
30,19\end{array}$ \\
\hline
\end{tabular}

Table I WC Configuration Results

To illustrate the influence of different number of WC for network performance, we deploy 4,8 and 12 WC respectively in the 48 nodes network by using the algorithm of subgraph + ADD optical backbone network configuration. The application of LG - FF routing policy is used for connection request routing and wavelength allocation. Each optical fiber is equipped with lambda number $\mathrm{W}=8$. The simulation under different number of WC in the configuration of network model and wavelength utilization rate is compared. Figure 4 shows that network congestion with the increase of the number of the WC. After the network configuration of four WC, the network performance is compared with that before the network WC configuration. The connectivity of network is better than that without wavelength conversion network connectivity. For the 12-WC network scenario, network connectivity to converge all over the wavelength conversion network.

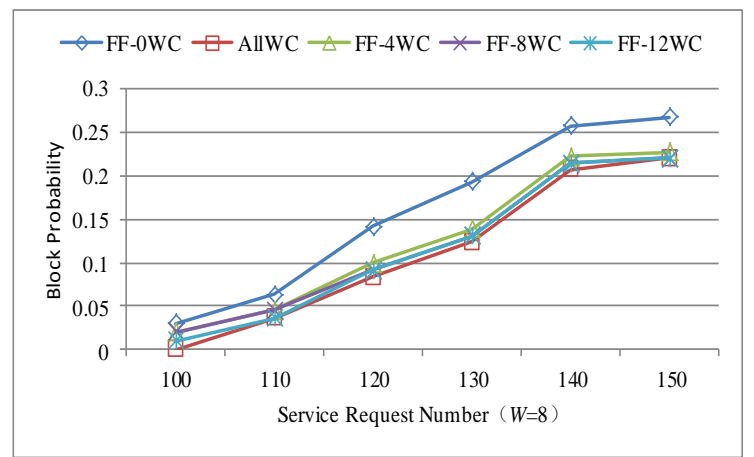

Fig.4 Block probability performance under different WC number

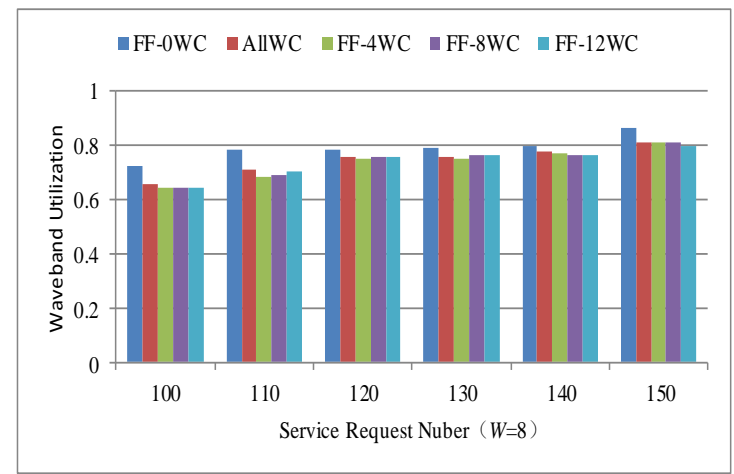

Fig.5 Block probability performance under different WC number 
Figure 5 shows that without the wavelength of wavelength conversion network usage, the largest network blocking is seen most commonly. As with the increasing number of service requests, the waveband utilization keep continuous increasing. Full wavelength conversion network can accommodate more connection requests, and works with the highest waveband utilization.

In order to analyze the computation speed between Sub_ADD and the proposed algorithm, we have compared the computation speed under different number of configured WCs (i.e., 12, 24, 36, 48). In general, each is directly proportional to the time consuming to configure the accuracy of the results of the algorithm, the result more accurate, the longer the calculation time, in the actual application to compromise should be considered. The detail simulations time for different algorithms are presented as in Table II, and we can conclude that the proposed algorithm experience slight faster computation speed.

\begin{tabular}{|l|c|c|c|c|}
\hline & 12 & 24 & 36 & 48 \\
\hline $\begin{array}{l}\text { Sub+ADD } \\
\text { /s }\end{array}$ & 27.863 & $\begin{array}{c}29.68 \\
4\end{array}$ & $\begin{array}{c}30.56 \\
8\end{array}$ & $\begin{array}{c}30.74 \\
2\end{array}$ \\
\hline $\begin{array}{l}\text { Proposed } \\
\begin{array}{l}\text { Algorithm } \\
\text { /s }\end{array}\end{array}$ & 27.428 & $\begin{array}{c}29.32 \\
8\end{array}$ & $\begin{array}{c}29.97 \\
2\end{array}$ & $\begin{array}{c}30.13 \\
6\end{array}$ \\
\hline
\end{tabular}

Table II Simulation Time Comparison for Different Algorithm

\section{Summary}

In this paper, we have presented a multi-layer routing wavelength assignment approach by using the subgraph algebraic decision diagram principle. The experimental results show that the proposed algorithm will slight increase the computation speed of the subgraph under multiple wavelength resource allocation. WC deployment can make the light channel without being limited by the wavelength of uniform continuity, and it can effectively reduce the network complexity. As the price of the WC is more expensive, the multi-layer approaches can accelerate the computation speed. Besides, the network performance under different WC deployment is presented.

\section{References}

[1] International Telecomunications Union, "ITU-T Recommendation G.694.1, Spectral grids for WDM applications: DWDM frequency grid", November 2012.

[2] M. Jinno et. al., "Spectrum-Efficient and Scalable Elastic Optical Path Network: Architecture, Benefits and Enabling Technologies", IEEE Comm. Mag., Nov. 2009, pp. 66-73.

[3] CHLAMTAC I, et al. Lightpath communications:an approach to high bandwidth opticalWANs[J].IEEE Trans Comm,1992,40(7):1165-1193.

[4] International Telecomunications Union, "ITU-T Recommendation G.694.1, Spectral grids for WDM applications: DWDM frequency grid", November 2012.

[5] M. Jinno et. al., "Spectrum-Efficient and Scalable Elastic Optical Path Network: Architecture, Benefits and Enabling Technologies", IEEE Comm. Mag., Nov. 2009, pp. 66-73. 